\title{
An Implementation Solution for Fractional Partial Differential Equations
}

\author{
Nicolas Bertrand, Jocelyn Sabatier, Olivier Briat, and Jean-Michel Vinassa \\ Bordeaux 1 University, CNRS UMR 5218, IMS Laboratory, 351 cours de la Libération, 33405 Talence Cedex, France \\ Correspondence should be addressed to Jocelyn Sabatier; jocelyn.sabatier@u-bordeauxl.fr
}

Received 9 November 2012; Accepted 3 December 2012

Academic Editor: José Tenreiro Machado

Copyright (C) 2013 Nicolas Bertrand et al. This is an open access article distributed under the Creative Commons Attribution License, which permits unrestricted use, distribution, and reproduction in any medium, provided the original work is properly cited.

The link between fractional differentiation and diffusion equation is used in this paper to propose a solution for the implementation of fractional diffusion equations. These equations permit us to take into account species anomalous diffusion at electrochemical interfaces, thus permitting an accurate modeling of batteries, ultracapacitors, and fuel cells. However, fractional diffusion equations are not addressed in most commercial software dedicated to partial differential equations simulation. The proposed solution is evaluated in an example.

\section{Introduction}

For an electrochemical system, species diffusion is often modeled by Fick's laws [1]. However, in almost all systems, the transport mechanism is different from the classical diffusion characterized by Fick's laws. This kind of diffusion is denoted by anomalous diffusion [2]. Anomalous diffusion is characterized by a mean squared displacement of the diffusing particles that has a power law dependence on time $\left\langle r^{2}\right\rangle \sim t^{\gamma}$ with $\gamma$ between 0 and 2 (for classical diffusion $\left\langle r^{2}\right\rangle \sim t$ ).

The theoretical approach of this type of diffusion is strongly related to fractional calculus [3]. It was indeed demonstrated that stochastic processes of random walks can be represented by fractional diffusion equations [4].

Among all existing anomalous diffusion equations, three diffusion modes, respectively, called "anomalous diffusion Ia" (ADIA), "anomalous diffusion Ib" (ADIB), and "anomalous diffusion II" (ADII) are characterized by a Fick's equations adaptation for phenomena considered [5].

In this paper, the authors have only considered ADIB type diffusion equations but the proposed contribution can be extended to others classes of equation. In the sequel, the link between fractional differentiation and diffusion is used to propose a solution for the implementation of a fractional diffusion equation in software such as COMSOL Multiphysics. These software applications are now powerful tools for engineers to simulate complex systems combining several physical domains such as electrochemistry and thermal. However they are not adapted to take into account anomalous diffusion and thus to model diffusion interfaces as in batteries, ultracapacitors, or fuel cells.

The link between fractional differentiation and diffusion equation is reminded in the second section of the paper. This link should be used to implement fractional differentiation in software dedicated to numerical solving of partial differential equation such as COMSOL Multiphysics software. However, as shown in Section 2, the diffusion equation form of a fractional system requires the computation of an inverse Fourier transform that is in most cases impossible to get analytically. This is why this paper proposes alternative partial differential equations approximation that exhibits a fractional behavior in a given frequency band. These differential equations can be easily implemented to simulate a fractional differentiator and thus a fractional diffusion equation.

\section{Link between Fractional Systems and Partial Differential Equations}

2.1. Partial Differential Equation Representation and Approximation of a Fractional System. For presentation simplicity, 


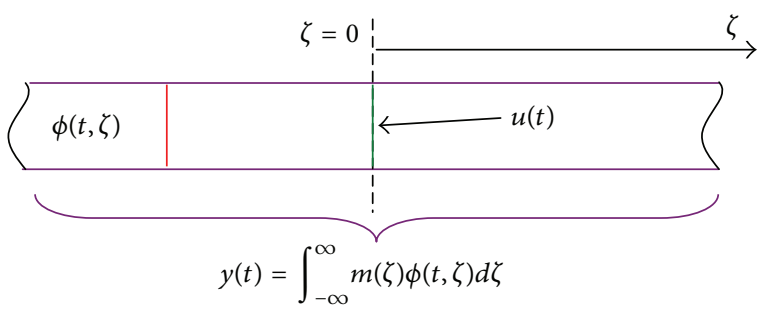

FIGURE 1: Representation of system (12) and thus system (1).

the following fractional system (fractional integrator) is considered

$$
H(s)=s^{-\gamma},
$$

with $0<\gamma<1$. Its link with diffusion equation can be demonstrated using the system impulse response [6] defined by the Mellin-Fourier integral of (1):

$$
h(t)=L^{-1}\left\{s^{-\gamma}\right\}=\lim _{\omega \rightarrow \infty} \frac{1}{2 j \pi} \int_{c-j \infty}^{c+j \infty} e^{s t} s^{-\gamma} d s,
$$

where $c$ is greater than the abscissa of the singular points of $H(s)$. Using poles definition that can be found in $[6,7]$, this system does not generate poles and its impulse response is thus given by

$$
h(t)=\frac{\sin (\gamma \pi)}{\pi} \int_{0}^{\infty} x^{-\gamma} e^{-t x} d x .
$$

Response of system (1) to an input $u(t)$ is defined as the convolution product of the impulse response $h(t)$ with the input $u(t)$ :

$$
y(t)=\int_{0}^{t} h(t-\tau) u(\tau) d \tau,
$$

and thus using relation (3) and through an integral permutation

$$
y(t)=\int_{0}^{\infty} \frac{\sin (\gamma \pi)}{\pi} x^{-\gamma}\left(\int_{0}^{t} e^{-(t-\tau) x} u(\tau) d \tau\right) d x .
$$

Let

$$
w(t, x)=\int_{0}^{t} e^{-(t-\tau) x} u(\tau) d \tau
$$

the following state space representation can be obtained for system (1):

$$
\begin{gathered}
\frac{\partial w(t, x)}{\partial t}=-x w(t, x)+u(t), \\
y(t)=\frac{\sin (\gamma \pi)}{\pi} \int_{0}^{\infty} x^{-\gamma} w(t, x) d x .
\end{gathered}
$$

Such a representation can be generalised to a large class of fractional systems as demonstrated in $[8,9]$. In these works, second relation in (7) is rewritten as

$$
y(t)=\int_{0}^{\infty} \mu(x) w(t, x) d x
$$

and representations (7) and (8) are denoted by diffusive representation. For a fractional transfer function defined by

$$
F(p)=\frac{B(p)}{A(p)}
$$

with $B(p)=\sum_{l=0}^{r} q_{l} p^{\beta_{l}}$ and $A(p)=\sum_{k=0}^{m} r_{k} p^{\alpha_{k}}$ where $\beta_{l+1} \geq$ $\beta_{l} \geq 0$, function $\mu(x)$ is defined by [9]

$$
\begin{aligned}
& \mu(x) \\
& \quad=\frac{1}{2 i \pi}\left[F\left((-x)^{-}\right)-F\left((-x)^{+}\right)\right] \\
& \quad=\frac{1}{\pi} \frac{\sum_{k=0}^{m} \sum_{l=0}^{q} a_{k} q_{l} \sin \left(\left(\alpha_{k}-\beta_{l}\right) \pi\right) x^{\alpha_{k}+\beta_{l}}}{\sum_{k=0}^{m} \alpha_{k}^{2} x^{2 \alpha_{k}}+\sum_{0 \leq k<l<m} 2 a_{k} a_{l} \cos \left(\left(\alpha_{k}-\alpha_{l}\right) \pi\right) x^{\alpha_{k}+\alpha_{l}} .}
\end{aligned}
$$

Initial conditions are defined for system (7) by $w(0, x)=$ $\rho(x)$ and thus permits giving the exact expression of the system response with initial conditions [7]

$$
y(t)=\int_{0}^{\infty} \mu(x)\left(w(0, x) e^{-x t}+\int_{0}^{t} e^{-x(t-\tau)} u(\tau) d \tau\right) d x
$$

Through several changes of variables described in [7], system (1), (but also a large number of fractional systems) can be described by

$$
\begin{gathered}
\frac{\partial \phi(t, \zeta)}{\partial t}=\frac{\partial^{2} \phi(t, \zeta)}{\partial \zeta^{2}}+u(t) \delta(\zeta), \\
y(t)=\int_{-\infty}^{\infty} m(\zeta) \phi(t, \zeta) d \zeta .
\end{gathered}
$$

Relation (10) shows that a fractional integrator can thus be seen as an infinite dimensional system described by a diffusion equation. This interpretation is represented by Figure 1 [7] in which

(i) the input $u(t)$ applied at the abscissa $\zeta=0$,

(ii) the real distributed state $\phi(t, \zeta)$,

(iii) the output (weighed sum on the state) appears. This remark can be generalized to a large number of fractional systems and thus demonstrates their link with diffusion equations.

Implementation of relation (10) requires the integral truncation that can be done as follows:

$$
y(t)=\int_{-\zeta_{2}}^{\zeta_{1}} m(\zeta) \phi(t, \zeta) d \zeta
$$

In (13) $m(\zeta)=\mathfrak{F}^{-1}\left\{4 \pi^{2} x \mu\left(4 \pi^{2} x^{2}\right)\right\}$ where $\mathfrak{F}^{-1}$ denotes the inverse Fourier transform. This relation is in practice impossible to compute analytically in most cases. To solve this problem, another partial differential equation is now proposed. 
2.2. Another Partial Differential Equation Approximation. Using Laplace transform and introducing $\mu(x)$ function $\left(\mu(x)=\sin (\gamma \pi) x^{-\gamma} / \pi\right.$ for a fractional integrator such as (1)), relation (3) becomes

$$
H(s)=\int_{0}^{+\infty} \frac{\mu(x)}{(s+x)} d x .
$$

Using change of variable $x=e^{-z}$, relation (15) becomes

$$
H(s)=\int_{-\infty}^{+\infty} \frac{\mu\left(e^{-z}\right) e^{-z}}{\left(s+e^{-z}\right)} d z .
$$

Implementation of such a transfer function requires the integral truncation, namely,

$$
H(s) \approx \int_{Z_{i}}^{Z_{f}} \frac{\mu\left(e^{-z}\right)}{\left(s / e^{-z}+1\right)} d z .
$$

Note that $Z_{i} \in \mathbb{R}, Z_{f} \in \mathbb{R}$ are homogenous to the logarithm of a frequency. Now Let $x(z, t)$ be a function of the space variable $z$ of finite dimension $\left(z \in\left\lfloor Z_{i} \cdots Z_{f}\right\rfloor\right)$ and of the time variable $t$. This function satisfies the class of partial differential equations

$$
\beta(z) \frac{\partial w(z, t)}{\partial z}+\gamma(z) \frac{\partial^{2} w(z, t)}{\partial t \partial z}=u(t)
$$

Also, let the system output $y(t)$ be given by

$$
y(t)=w\left(Z_{f}, t\right)-w\left(Z_{i}, t\right)=\int_{Z_{i}}^{Z_{f}} \frac{\partial w(z, t)}{\partial z} d z .
$$

This partial differential equation class has been studied in [10]. Transfer function that links the system input and output is defined by

$$
G(s)=\frac{Y(s)}{U(s)}=\int_{0}^{Z} \frac{\beta^{-1}(z)}{1+\gamma(z) \beta^{-1}(z) s} d z .
$$

Now if

$$
\begin{gathered}
\beta^{-1}(z)=\mu\left(e^{-z}\right), \\
\gamma^{-1}(z) \beta(z)=e^{-z} \text { and thus } \gamma(z)=\frac{e^{z}}{\mu\left(e^{-z}\right)} .
\end{gathered}
$$

It turns out that the solution of the partial differential equation defined by (15) and (16) is an approximation of the response of the system whose transfer is defined by (14) (and thus by (1) for a fractional integrator). Such a representation can thus be used to approximate a fractional system or a fractional integrator. It is now used to implement a fractional diffusion equation.

\section{Application to Fractional Partial Differential Equations Implementation}

Implementation problem of a fractional partial differential equation using simulation software such as Comsol Multiphysics is now addressed and the followingclass of equation is considered:

$$
\frac{\partial^{1-\gamma} C_{1}(t, x)}{\partial t^{1-\gamma}}=-D \frac{\partial^{2} C_{1}(t, x)}{\partial x^{2}}
$$

with the following boundary and initial conditions:

$$
\frac{\partial C_{1}(t, 0)}{\partial x}=\frac{\partial C_{1}(t, L)}{\partial x}=0, \quad C_{1}(0, x)=g(x) .
$$

It is an $A D I b$ type anomalous diffusion equation that can also be rewritten as

$$
Y(t, x)=-D \frac{\partial^{2} C_{1}(t, x)}{\partial x^{2}}
$$

with

$$
\begin{aligned}
Y(t, x) & =\frac{\partial^{1-\gamma} C_{1}(t, x)}{\partial t^{1-\gamma}} \\
\frac{\partial C_{1}(t, 0)}{\partial x}=\frac{\partial C_{1}(t, L)}{\partial x} & =0, \quad C_{1}(0, x)=f(x) .
\end{aligned}
$$

According to the demonstration in Section 2 and in [11], (1$\gamma$ ) fractional derivative of $C_{1}(t, x)$ can be approximated by

$$
\begin{gathered}
U(t, x)=\frac{\partial C_{1}(t, x)}{\partial t} \\
U(t, x)=\frac{\partial w(z, x, t)}{\partial z} \frac{1}{R(z)}+C(z) \frac{\partial^{2} w(z, x, t)}{\partial z \partial t} \\
Y(t, x)=w\left(Z_{f}, x, t\right)-w\left(Z_{i}, x, t\right)=\int_{Z_{i}}^{Z_{f}} \frac{\partial w(z, x, t)}{\partial z} d z
\end{gathered}
$$

where functions $C(z)$ and $R(z)$ are, respectively, defined by

$$
C(z)=C(0) e^{-A z}, \quad R(z)=R(0) e^{B z}
$$

Implementation of (24) using approximations from (26) to (29) thus requires 2 geometries. In a first 1D-type geometry, (23) is implemented. To take into account relation (24), a second 2D-type geometry is created.

Values $Z_{i}$ and $Z_{f}$ for the $2 \mathrm{D}$ geometry along the $z$ axis are used to define the range of frequency $\left[\omega_{i}, \omega_{f}\right]$ for which the approximation of the fractional differentiation is expected using the relations

$$
\omega_{i}=e^{Z_{i}}, \quad \omega_{f}=e^{Z_{f}} .
$$

Note that frequencies $\omega_{i}$ and $\omega_{f}$ depend, respectively, on the simulation duration $T_{d}$ and the sampling time $T_{s}$. These frequencies can be defined using the following rules: $\omega_{i} \ll$ $2 \pi / T_{d}$ and $\omega_{f} \gg 2 \pi / T_{s}$. 


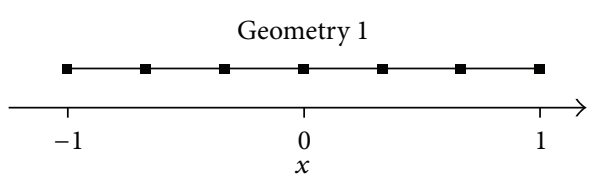

FIGURE 2: 1D geometry representation.

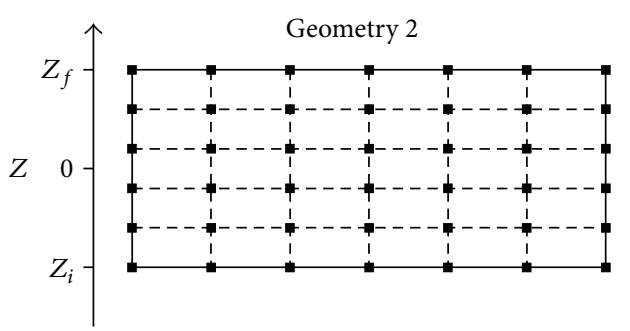

FIGURE 3: 2D geometry representation.

Information produced in the two geometries are then exchanged as described in Figure 4.

\section{Example}

The following diffusion system with $x \in[0 \cdots L]$ is considered:

$$
\frac{\partial^{1-\gamma} C_{1}(t, x)}{\partial t^{1-\gamma}}=-D \frac{\partial^{2} C_{1}(t, x)}{\partial x^{2}}
$$

with the following initial and boundaries conditions:

$$
\begin{gathered}
C_{1}(t, 0)=h(t), \quad C_{1}(t, L)=j(t), \\
C_{1}(0, x)=0 .
\end{gathered}
$$

For $\gamma=0.5, D=0.1 \mathrm{~m} / \mathrm{s}, j(t)=0$ and

$$
\begin{aligned}
h(t)= & K \cdot\left(t-t_{0}\right) \text { heaviside }\left(t-t_{0}\right) \\
& -K\left(t-t_{1}\right) \text { heaviside }\left(t-t_{1}\right) \\
= & h_{1}(t)-h_{2}(t) \quad t \geq 0 .
\end{aligned}
$$

As shown in the appendix, system (31) and (32) solution is defined by

$$
C_{1}(x, t)=0 \text { for } t \leq t_{0}
$$

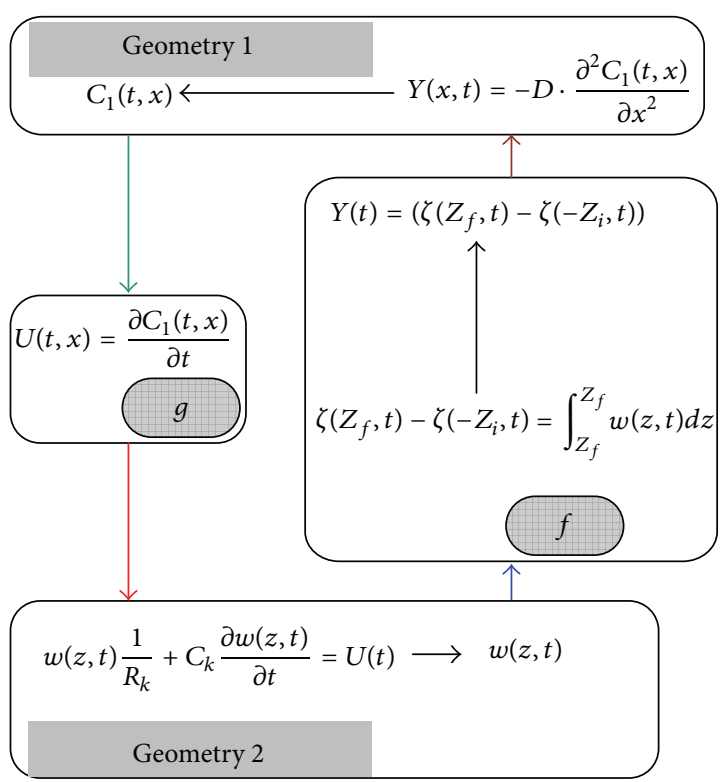

FIGURE 4: Information exchanged between the two geometries.

$C_{1}(x, t)$

$=\sum_{0}^{\infty}\left(\begin{array}{l}2 \cdot \frac{\left(1-e^{(n \pi)^{4} D^{2}\left(t-t_{0}\right)} \operatorname{erfc}\left((n \pi)^{2} D \sqrt{\left(t-t_{0}\right)}\right)\right)}{(n \pi)^{5} D^{2}} \\ -\frac{4 \cdot\left(t-t_{0}\right)^{0.5}}{(n \pi)^{3} D \cdot \Gamma(0.5)}\end{array}\right)$

$\times \sin (n \pi x)+(1-x) \cdot h_{1}(t) \quad$ for $t_{0} \leq t \leq t_{1}$,

$C_{1}(x, t)$

$$
\begin{aligned}
& =\sum_{0}^{\infty}\left(\begin{array}{c}
2 \cdot \frac{\left(1-e^{(n \pi)^{4} D^{2}\left(t-t_{0}\right)} \operatorname{erfc}\left((n \pi)^{2} D \sqrt{\left(t-t_{0}\right)}\right)\right)}{(n \pi)^{5} D^{2}} \\
-2 \cdot \frac{4 \cdot\left(t-t_{0}\right)^{0.5}}{(n \pi)^{3} D \cdot \Gamma(0.5)} \\
+\frac{4 \cdot\left(t-t_{1}\right)^{0.5}}{(n \pi)^{3} D \cdot \Gamma(0.5)}
\end{array}\right) \\
& \times \sin (n \pi x)+(1-x) \cdot\left(h_{1}(t)-h_{2}(t)\right) \quad \text { for } t \geq t_{1} \text {. }
\end{aligned}
$$

For $t_{1}=5 \mathrm{~s}$ and $t_{2}=10 \mathrm{~s}$, this solution is represented by Figure 5.

The analytical solution is compared with the results produced by COMSOL Multiphysics for function $C_{1}(x, t)$ using the implementation and geometries described in Figures 2-4. For the implementation, $\omega_{i}=0.01 \mathrm{rd} / \mathrm{s}$ and $\omega_{f}=1000 \mathrm{rd} / \mathrm{s}$. 


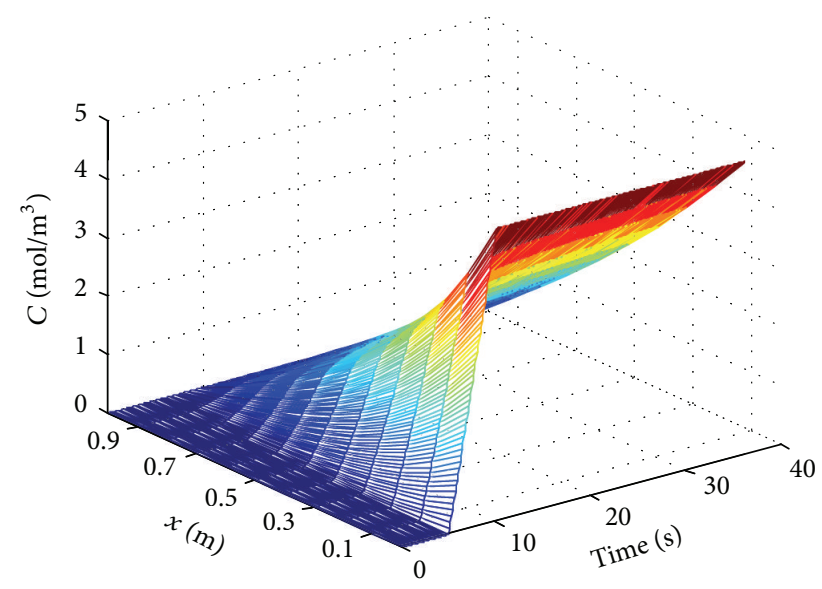

FIgURE 5: Function $C_{1}(x, t)$ produced by COMSOL Multiphysics.

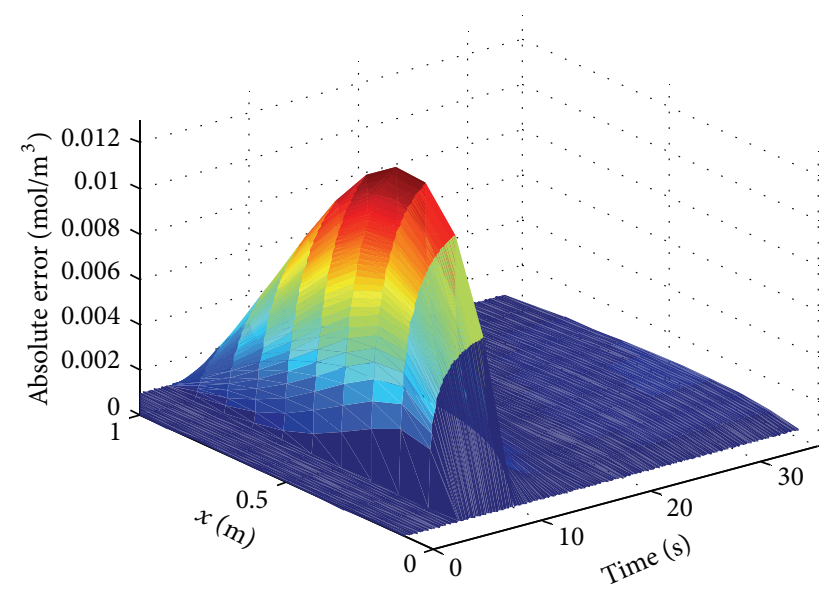

Figure 6: Absolute error between function $C_{1}(x, t)$ and the solution computed with COMSOL Multiphysics.

Figure 6 is a representation of the absolute error between function $C_{1}(x, t)$ and the solution computed with COMSOL Multiphysics. This really small error permits us to validate the method we used for the implementation of a fractional diffusion equation using a partial differential equation for the approximation of a fractional derivative.

\section{Conclusion}

This paper proposes a method for the implementation of a fractional diffusion equation into simulation softwares such as COMSOL Multiphysics. These software applications are now powerful tools for engineers to simulate complex systems combining several physical domains such as electrochemical and thermal. However they are not adapted to take into account anomalous diffusion and thus to model diffusion interfaces as in batteries, ultracapacitors, or fuel cells. To permit the implementation, the link between fractional systems and diffusion equation is used. The fractional diffusion equation considered is splitted into two parts and the remaining fractional equation is approximated by a partial differential equation. For the implementation of this partial differential equation, an additional geometry is created (a $1 \mathrm{D}$ system is transformed into a $2 \mathrm{D}$ system). The efficiency of the proposed method is evaluated in an example. The results obtained showed the efficiency of the proposed method.

\section{Appendix}

This appendix demonstrates how, using material provided in [12], the analytical solution of the following fractional diffusion equation:

$$
\frac{\partial^{1-\gamma} C_{1}(t, x)}{\partial t^{1-\gamma}}=-D \frac{\partial^{2} C_{1}(t, x)}{\partial x^{2}}, \quad x \in[0 \cdots L]
$$

with the following initial and boundaries conditions

$$
C_{1}(t, 0)=h(t), \quad C_{1}(t, L)=j(t), \quad C_{1}(0, x)=0,
$$

is obtained. To obtain homogenous conditions at $x=0$ and $x=1$, the following change of variable is used:

$$
V(x, t)=C_{1}(x, t)+U(x, t)
$$

with

$$
U(x, t)=\left(1-\frac{x}{L}\right) h(t)+\frac{x}{L} j(t) .
$$

System (A.1) thus becomes

$$
\begin{gathered}
\frac{\partial^{1-\gamma} V(t, x)}{\partial t^{1-\gamma}}=-D \frac{\partial^{2} V(t, x)}{\partial x^{2}}+f(x, t), \\
V(t, 0)=0, \quad V(L)=0, \quad V(0, x)=g(x),
\end{gathered}
$$

with

$$
\begin{gathered}
f(x, t)=-\left(1-\frac{x}{L}\right) \frac{\partial^{1-\gamma} h(t)}{\partial t^{1-\gamma}}-\frac{x}{L} \frac{\partial^{1-\gamma} j(t)}{\partial t^{1-\gamma}}, \\
g(x)=-\left(1-\frac{x}{L}\right) h(0)-\frac{x}{L} j(0) .
\end{gathered}
$$

Separation variable method leads to writing $V(x, t)$ as:

$$
V(x, t)=\sum_{1}^{\infty} T_{n}(t) \sin (n \pi x)
$$

with

$$
\begin{gathered}
\frac{\partial^{1-\gamma} T_{n}(t)}{\partial t^{1-\gamma}}+(n \pi)^{2} D T_{n}(t)=f_{n}(t), \\
T_{n}(0)=2 \int_{0}^{1} g(\zeta) \sin (n \pi \zeta) d \zeta, \\
f_{n}(t)=2 \int_{0}^{1} f(x, t) \sin (n \pi x) d x .
\end{gathered}
$$


Now let $\gamma=0.5, j(t)=0$, and

$$
\begin{gathered}
h(t)=0, \quad t \leq t_{0}, \\
h(t)=K\left(t-t_{0}\right), \quad t \leq t \leq t_{1}, \\
h(t)=K\left(t_{1}-t_{0}\right), \quad x \geq t_{1} .
\end{gathered}
$$

Solution of system (A.1) is given, according to (A.9), (A.10), and (A.11)

$$
\begin{gathered}
V(x, t)=\sum_{1}^{\infty} T_{n}(t) \sin (n \pi x), \\
\frac{\partial^{1-\gamma} T_{n}(t)}{\partial t^{1-\gamma}}+(n \pi)^{2} D T_{n}(t)=f_{n}(t), \\
T_{n}(0)=0
\end{gathered}
$$

with

$$
\begin{gathered}
f_{n}(t)=2 \int_{0}^{1} f(x, t) \sin (n \pi x) d x, \\
f(x, t)=-(1-x) \frac{\partial^{1-\gamma} h(t)}{\partial t^{1-\gamma}}, \\
g(x)=-(1-x) h(0)=0, \\
V(x, t)=C_{1}(x, t)+U(x, t), \\
U(x, t)=(1-x) h(t) .
\end{gathered}
$$

Combining relations (A.19) and (A.20) leads to

$$
f_{n}(t)=-2 \frac{\partial^{1-\gamma} h(t)}{\partial t^{1-\gamma}} \int_{0}^{1}(1-x) \sin (n \pi x) d x,
$$

and thus

$$
f_{n}(t)=-\frac{4}{n \pi} \frac{\partial^{1-\gamma} h(t)}{\partial t^{1-\gamma}} .
$$

Laplace transform applied to (A.17) leads to

$$
p^{0.5} T_{n}(p)+(n \pi)^{2} D T_{n}(p)=\frac{4}{n \pi} p^{0.5} h(p),
$$

and thus

$$
T_{n}(p)=\frac{(4 / n \pi) p^{0.5} h(p)}{p^{0.5}+(n \pi)^{2} D} .
$$

If $h(t)$ is written as

$$
\begin{aligned}
h(t)= & K\left(t-t_{0}\right) \text { heaviside }\left(t-t_{0}\right) \\
& -K\left(t-t_{1}\right) \text { heaviside }\left(t-t_{1}\right) \\
= & h_{1}(t)-h_{2}(t), \quad t \geq 0,
\end{aligned}
$$

relation (A.27) becomes

$$
\begin{aligned}
T_{n}(p) & =\frac{(4 / n \pi) p^{0.5} h_{1}(p)}{p^{0.5}+(n \pi)^{2} D}-\frac{(4 / n \pi) p^{0.5} h_{2}(p)}{p^{0.5}+(n \pi)^{2} D} \\
& =T_{n 1}(p)-T_{n 2}(p) .
\end{aligned}
$$

Within the interval $0<t<t_{0}, h_{1}(t)=0$ and thus using (A.27) $T_{n 1}(t)=0$.

If $t>t_{0}$, function $h(t)$ is a ramp and thus

$$
T_{n 1}(p)=\frac{(4 / n \pi) p^{0.5}\left(K / p^{2}\right)}{p^{0.5}+(n \pi)^{2} D} .
$$

Inverse Laplace transform then permits

$$
\begin{aligned}
T_{n 1}(t)= & 2 \cdot \frac{\left(1-e^{(n \pi)^{4} D^{2}\left(t-t_{0}\right)} \operatorname{erfc}\left((n \pi)^{2} D \sqrt{\left(t-t_{0}\right)}\right)\right)}{(n \pi)^{5} D^{2}} \\
& -\frac{4 \cdot\left(t-t_{0}\right)^{0.5}}{(n \pi)^{3} D \cdot \Gamma(0.5)} .
\end{aligned}
$$

Within the interval $0<t<t_{1}, h_{2}(t)=0$, and thus $T_{n 2}(t)=0$.

Using a similar method, $T_{n 2}(t)$ where $t>t_{1}$ is given by

$$
\begin{aligned}
T_{n 2}(t)= & 2 \cdot \frac{\left(1-e^{(n \pi)^{4} D^{2}\left(t-t_{1}\right)} \operatorname{erfc}\left((n \pi)^{2} D \sqrt{\left(t-t_{1}\right)}\right)\right)}{(n \pi)^{5} D^{2}} \\
& -\frac{4 \cdot\left(t-t_{1}\right)^{0.5}}{(n \pi)^{3} D \cdot \Gamma(0.5)} .
\end{aligned}
$$

Finally, using (A.3), system (A.1) and (A.2) solution is defined by, using (34), (35), and (36).

\section{Conflict of Interests}

The authors declare no conflict of interests.

\section{References}

[1] R. B. Bird, W. E. Stewart, and E. N. Lightfoot, Transport Phenomena, John Wiley \& Sons, New York, NY, USA, 2nd edition, 2002.

[2] A. Compte and R. Metzler, "The generalized Cattaneo equation for the description of anomalous transport processes," Journal of Physics A, vol. 30, no. 21, pp. 7277-7289, 1997.

[3] A. Compte, "Stochastic foundations of fractional dynamics," Physical Review E, vol. 53, no. 4, pp. 4191-4193, 1996.

[4] R. Gorenflo and F. Mainardi, "Some recent advances in theory and simulation of fractional diffusion processes," Journal of Computational and Applied Mathematics, vol. 229, no. 2, pp. 400-415, 2009.

[5] J. Bisquert and A. Compte, "Theory of the electrochemical impedance of anomalous diffusion," Journal of Electroanalytical Chemistry, vol. 499, no. 1, pp. 112-120, 2001.

[6] A. Oustaloup, Systèmes Asservis Linéaires D’ordre Fractionnaire, Masson, Paris, France, 1983.

[7] J. Sabatier, M. Merveillaut, R. Malti, and A. Oustaloup, "How to impose physically coherent initial conditions to a fractional system?" Communications in Nonlinear Science and Numerical Simulation, vol. 15, no. 5, pp. 1318-1326, 2010.

[8] G. Montseny, "Diffusive representation of pseudo-differential time-operators," Esaim, vol. 5, pp. 159-175, 1998. 
[9] D. Matignon, "Stability properties for generalized fractional differential systems," Esaim, vol. 5, pp. 145-158, 1998.

[10] F. Levron, J. Sabatier, A. Oustaloup, and L. Habsieger, "From partial differential equations of propagative recursive systems to non-integer differentiation," Fractional Calculus \& Applied Analysis, vol. 2, no. 3, pp. 245-264, 1999.

[11] J. Sabatier, H. C. Nguyen, X. Moreau, and A. Oustaloup, "Fractional behaviour of partial differential equations whose coefficients are exponential functions of the space variable," Mathematical and Computer Modelling of Dynamical Systems, 2013.

[12] Y. Lin and C. Xu, "Finite difference/spectral approximations for the time-fractional diffusion equation," Journal of Computational Physics, vol. 225, no. 2, pp. 1533-1552, 2007. 


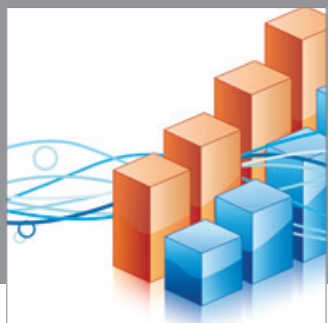

Advances in

Operations Research

mansans

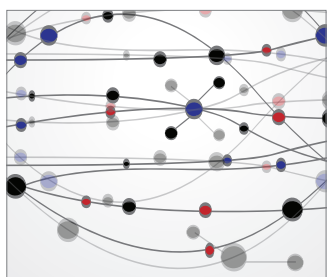

The Scientific World Journal
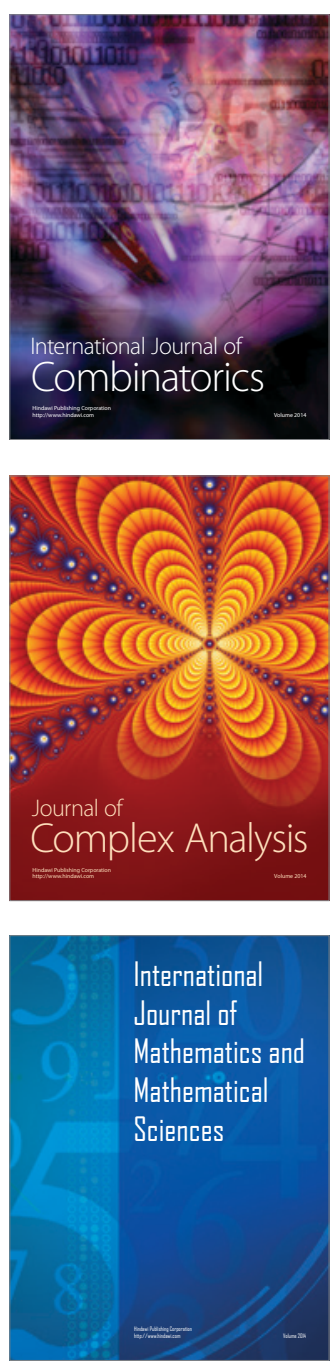
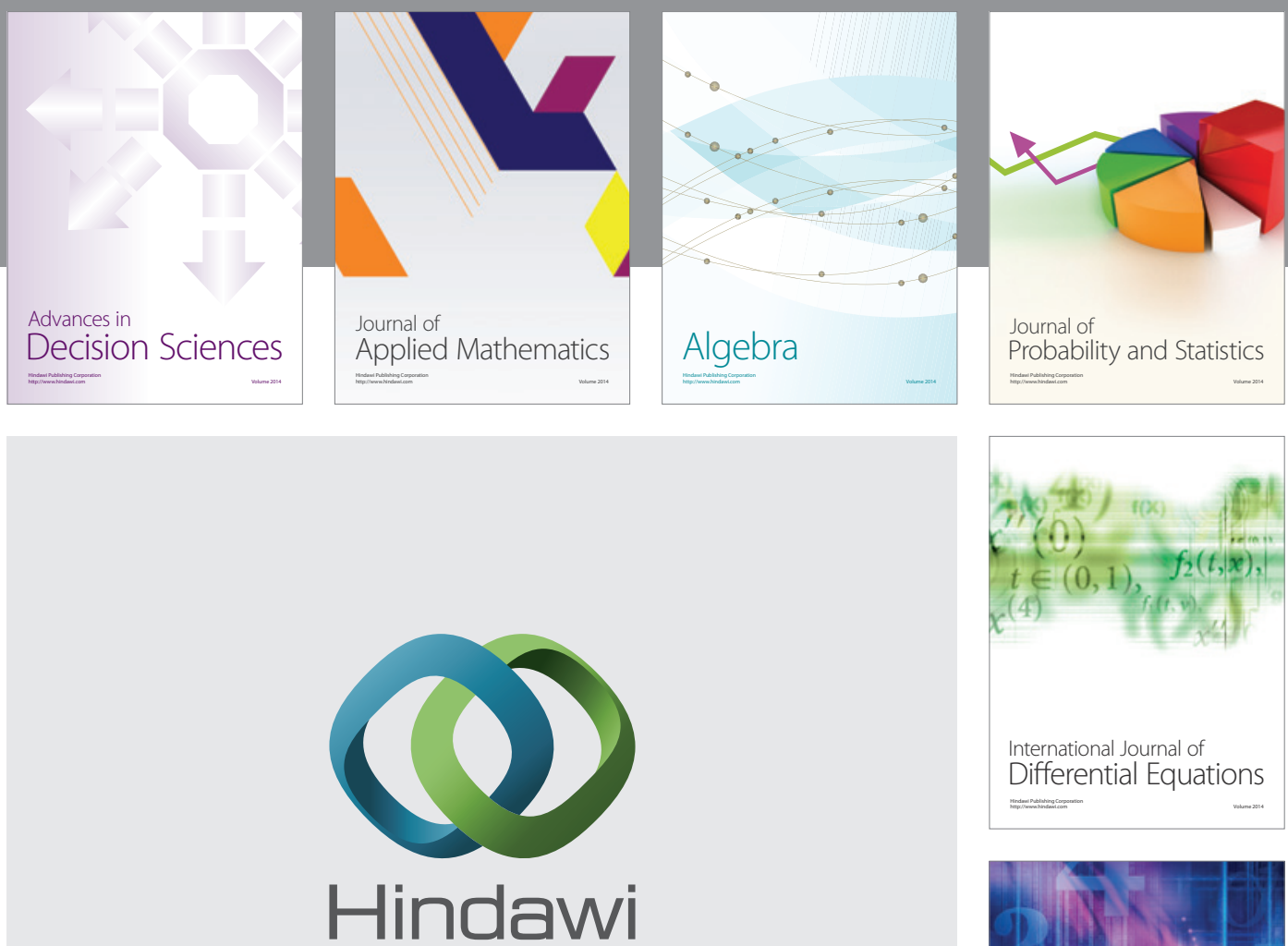

Submit your manuscripts at http://www.hindawi.com
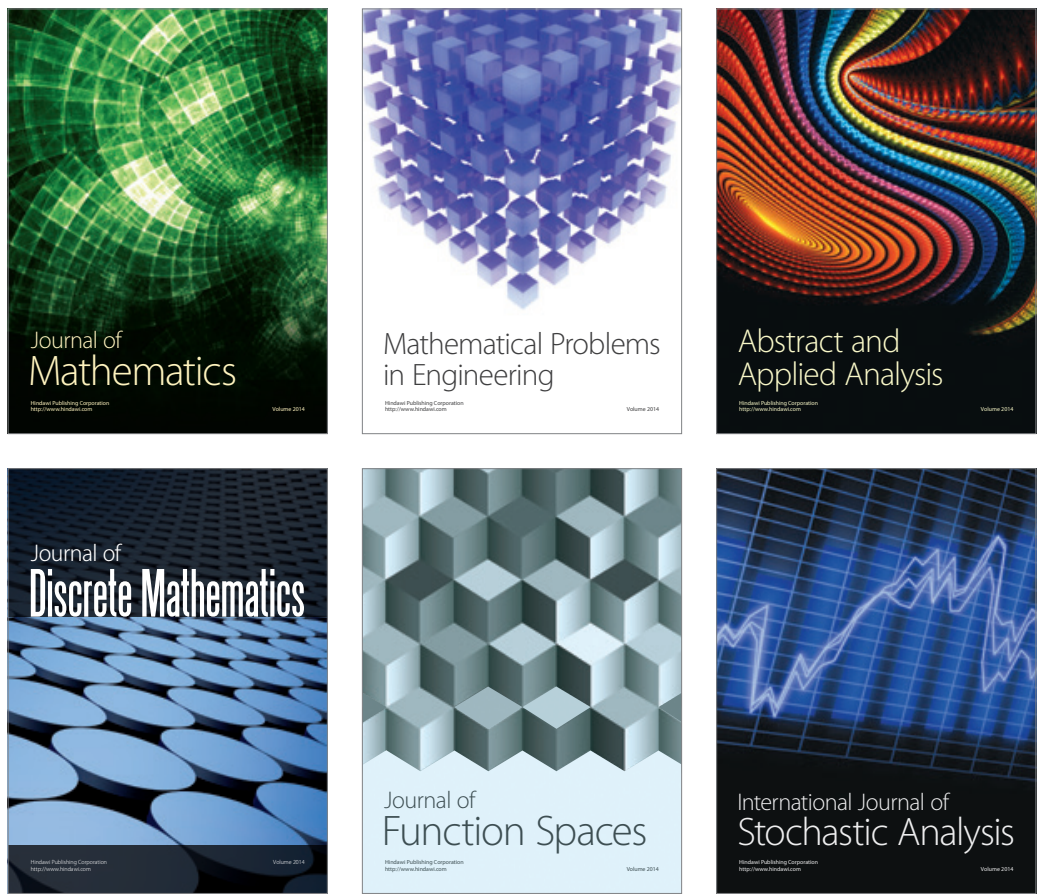

Journal of

Function Spaces

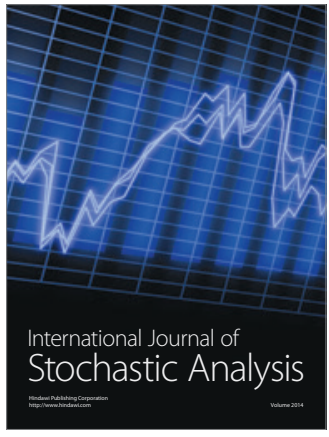

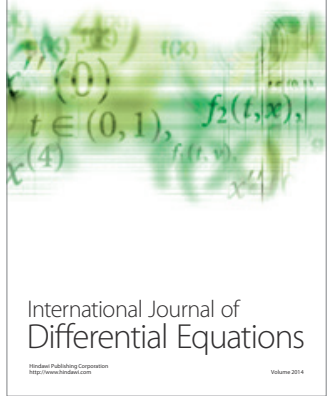
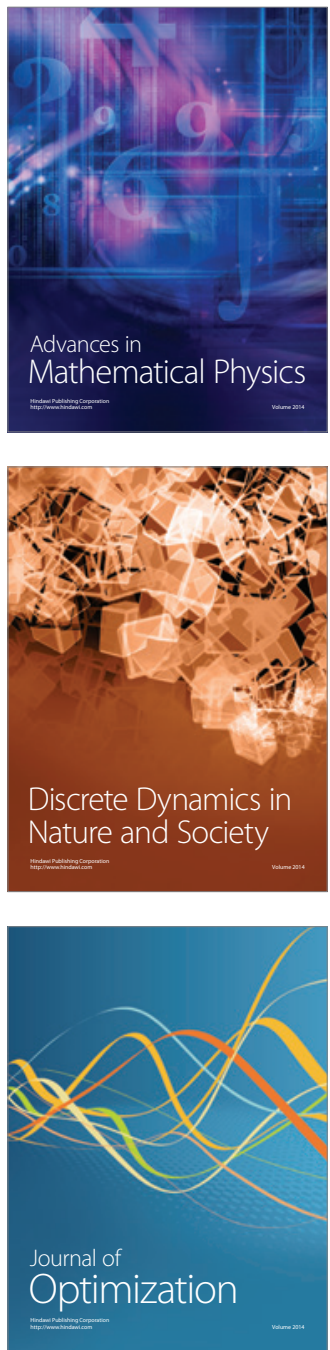